\title{
Perfusion Magnetic Resonance Imaging in Transient Symptoms of Lateral Medullary Syndrome
}

\author{
Hye Yoon Chung*, Bang-Hoon Cho*, Kyung-Yul Lee ${ }^{\star}, \dagger$ \\ Department of Neurology, Gangnam Severance Hospital, Yonsei University College of Medicine*, Seoul; Severance Institute for Vascular and \\ Metabolic Research, Yonsei University College of Medicine ${ }^{\dagger}$, Seoul, Korea
}

There were case reports of patients who had transient medullary symptoms but showed normal diffusion weighted imaging. However, there have been no reports yet concerning perfusion weighted imaging in such cases. We present two cases of patients had transient symptoms of lateral medullary syndrome. Initial diffusion and perfusion weighted imaging was obtained while the patients still had the symptoms. Brain magnetic resonance imaging showed perfusion delay in the corresponding regions, while diffusion weighted image showed negative findings. These cases suggest that perfusion weighted imaging might be helpful in diagnosing diffusion-negative lateral medullary syndrome.

\section{J Neurosonol Neuroimag 201\&;10(2):151-153}

Key Words: Lateral medullary syndrome; Perfusion magnetic resonance imaging; Magnetic resonance imaging; Transient ischemic attack
Received: July 27, 2018

Revised: September 4, 2018

Accepted: September 5, 2018

Address for correspondence: Kyung-Yul Lee

Department of Neurology, Gangnam Severance Hospital, Yonsei University College of Medicine, 211 Eonju-ro, Gangnam-gu, Seoul o6273, Korea

Tel: $+82-2-2019-3325$

Fax: $+82-2-3462-5904$

E-mail: kylee@yuhs.ac
Lateral medullary infarction is generally diagnosed from the clinical symptoms and signs, as well as magnetic resonance imaging (MRI) findings. ${ }^{1}$ To date, there have been reports using diffusion-weighted imaging (DWI) or both DWI and perfusion-weighted imaging (PWI) to evaluate medullary infarctions. ${ }^{2,3}$ There is evidence of perfusion MRI having diagnostic value in patients with transient ischemic attack (TIA) who present with speech impairment, sensory impairment, and hemiparesis but had normal DWI findings. ${ }^{4}$ However, there have been no reports where only perfusion delay on PWI without ischemic change correlated with lateral medullary syndrome. Here, we describe two patients who showed symptoms of lateral medullary syndrome without any lesions on DWI while perfusion delay was found in the corresponding area.

\section{CASE REPORT}

\section{Case 1}

A 32-year-old male was admitted for right-sided ptosis and rightward sway. He also complained of vertigo, nausea, and headache. On neurological examination, he showed right-sided ptosis and dysarthria. The initial National Institutes of Health Stroke Scale (NIHSS) score was 2. He had no other medical history except current cigarette smoking. He arrived at the hospital one hour after symptom onset and received a tissue plasminogen activator (tPA) infusion after brain computed tomography (CT) angiography. CT angiography showed non-visualization of the right distal vertebral artery which suggested arterial occlusion or severe stenosis (Fig. 1A). He recovered well, with no neurological sequelae two hours after tPA infusion.

Brain MRI was obtained 2 hours and 4 days after symptom onset. The initial DWI was obtained while the patient still had symptoms but did not show any 
ischemic change (Fig. 1B). However, the $\mathrm{T}_{\max }$ (Fig. ${ }_{1} \mathrm{C}$ ), mean transit time, and time to peak PWI indicated perfusion delay in the right lateral medulla area. The follow-up DWI showed no ischemic lesions and magnetic resonance angiography (MRA) showed mild stenosis of the right distal vertebral artery, which was better than initial CT angiography (Fig. 1D). One-year follow-up MRI showed no perfusion delay on PWI (Fig. $1 E$ ) and MRA showed resolved right vertebral artery stenosis (Fig. 1 F). Considering the fact that the vertebral artery stenosis was normalized within a relatively short period of time and that the posterior neck pain complained from the beginning, the right vertebral artery stenosis was presumed to be due to arterial dissection.

\section{Case 2}

An 82-year-old male was admitted for sudden vertigo and leftward sway. Right-beating horizontal spontaneous nystagmus was noted in neurological examination. There was no limb ataxia although left-sided falling tendency. The initial NIHSS score was o. He had a history of coronary artery occlusive disease with stent insertion and had been taking an antiplatelet agent. He had no other medical history. His vertiginous symptoms lasted only a few minutes but left-sided gait sway lasted 24 hours.

A brain MRI was obtained 18 hours after symptom onset, with no apparent ischemic lesion on DWI (Fig. 2A). However, the $\mathrm{T}_{\max }$ (Fig. $2 \mathrm{~B}$ ), mean transit time, and
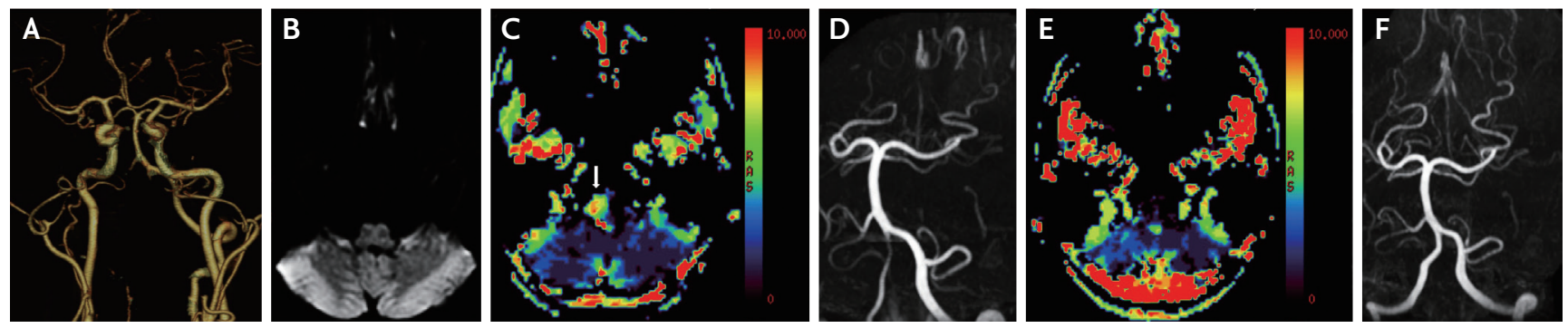

FIG. 1. Brain computed tomography (CT) angiography, magnetic resonance imaging and magnetic resonance angiography (MRA) of a 32-year-old man. (A) CT angiography shows non-visualization of the right distal vertebral artery suggesting severe stenosis or occlusion. (B) Initial diffusion weighted imaging shows no diffusion restriction in the right medulla. (C) Initial $\mathrm{T}_{\max }$ perfusion weighted image (PWI) shows perfusion delay in the right lateral medulla (arrow). (D) MRA performed 4 days after symptom onset shows visualization of the right distal vertebral artery with remaining stenosis. (E) One-year follow up PWI shows no perfusion delay. (F) One-year follow up MRA shows resolved right vertebral artery stenosis.
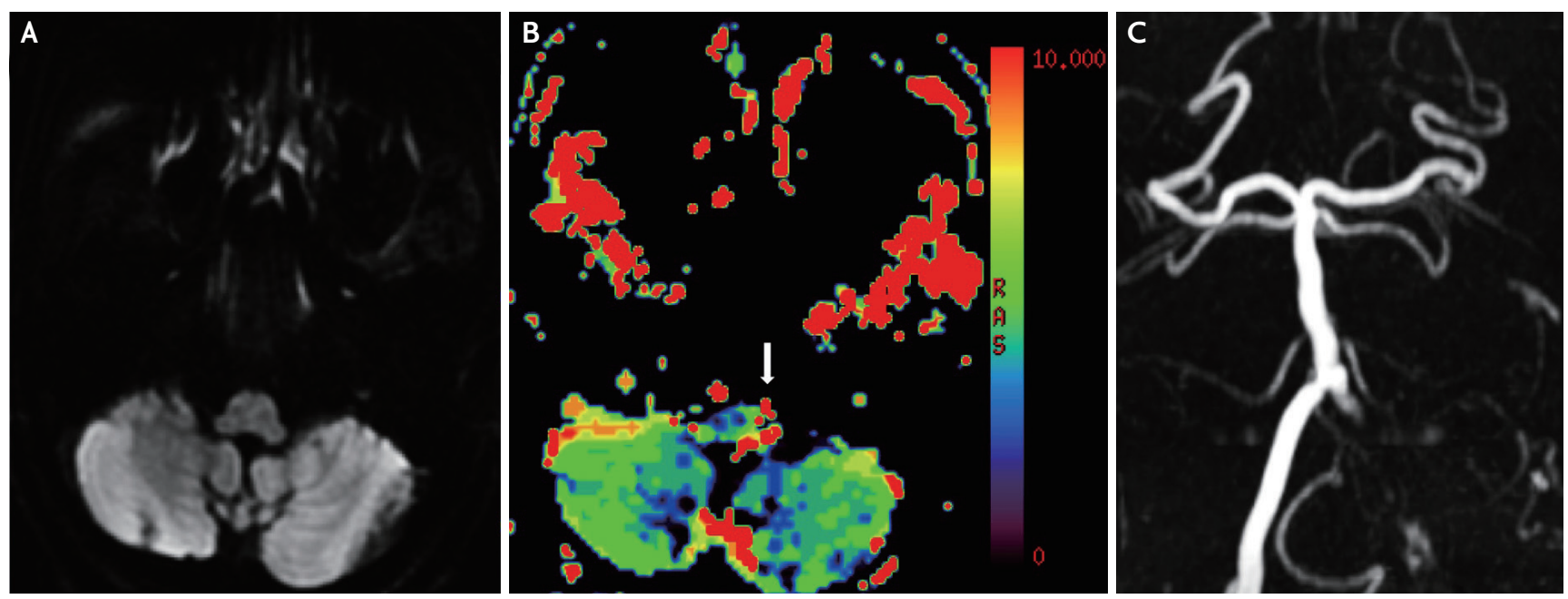

FIG. 2. Magnetic resonance imaging and magnetic resonance angiography (MRA) of an 82-year-old man. (A) Brain diffusion weighted imaging shows no diffusion restriction in the left medulla. (B) $T_{\text {max }}$ perfusion weighted imaging shows perfusion delay in the left lateral medulla (arrow). (C) MRA shows left distal vertebral artery occlusion. 
time to peak PWI indicated perfusion delay in the left medulla. MRA showed occlusion of the left distal vertebral artery (Fig. 2C). He was discharged without any symptoms the day after hospitalization.

\section{DISCUSSION}

Lateral medullary infarction arises when the posterior inferior cerebellar artery is compromised. The symptoms of lateral medullary infarction are ipsilateral facial sensory disturbance, contralateral body sensory disturbance, ipsilateral ataxia, ipsilateral Horner syndrome, and ipsi- or contralesional nystagmus can be found in some as well. ${ }^{5}$ So, in second case of this report, contralesional nystagmus and increased ipsilesional falling tendency could be explained by the involvement of vestibular nucleus and dorsal spinocerebellar tract or descending vestibulospinal tract, respectively. Important risk factors for lateral medullary infarction are vertebral artery dissection for younger patients and large artery atherosclerosis in older patients. ${ }^{6}$

A meta-analysis study indicates that DWI may be negative in as much as $6.8 \%$ of all acute ischemic stroke. ${ }^{7}$ The sensitivity of DWI for lateral medullary and lateral pontine infarctions is especially low, reported as $72 \%$ within the first 48 hours after onset in one study. ${ }^{8}$ Therefore, it has been suggested that in the first 72 hours after symptom onset, lateral medullary infarction should not be ruled out regardless of negative diffusion results. ${ }^{9}$

In our first case, a follow-up MRI obtained 4 days after symptom onset showed negative diffusion results, suggesting a TIA or resolved ischemic infarction due to tPA use. In our second case, the patient may have had TIA based on the negative DWI findings, but since no follow-up MRI was performed after the initial assessment, a lateral medullary infarction with early negative DWI findings cannot be ruled out. In both cases, cerebral blood volume and flow PWI revealed no significant abnormalities. Although the medullary lesion is too small to evaluate clearly on cerebral blood volume and flow PWI, preserved cerebral blood volume and flow may be the cause of the transient symptom despite of delayed time index of PWI.

In both cases, the patients displayed symptoms suggesting lateral medullary infarction and perfusion delay was seen in the lateral medulla despite negative DWI findings. Furthermore, both had a steno-occlusive lesion of the corresponding vertebral artery. Our report suggests that PWI can be useful in diagnosing diffusion-negative lateral medullary syndrome, especially in patients with mild and transient symptoms. However, we have to consider a limitation of PWI in detecting small lesion due to the limited spatial resolution and its susceptibility to motion artifacts.

\section{Conflicts of Interest}

No potential conflict of interest relevant to this article was reported.

\section{REFERENCES}

1. Kim JS. Pure lateral medullary infarction: clinical-radiological correlation of 130 acute, consecutive patients. Brain. 2003;126(Pt 8):1864-1872.

2. Kitis O, Calli C, Yunten N, Kocaman A, Sirin H. Wallenberg's lateral medullary syndrome: diffusion-weighted imaging findings. Acta Radiol. 2004;45:78-84.

3. Kim SJ, Ryoo S, Bang OY, Chung CS, Lee KH, Kim GM. Perfusion-weighted MRI as a predictor of clinical outcomes following medullary infarctions. Cerebrovasc Dis. 2010;29:382-388.

4. Grams RW, Kidwell CS, Doshi AH, Drake K, Becker J, Coull $\mathrm{BM}$, et al. Tissue-negative transient ischemic attack: is there a role for perfusion MRI? AJR Am J Roentgenol. 2016;207:157-162.

5. Baugh CW, Brown DF, Nadel ES. Horner's syndrome, hoarseness, and unsteady gait. J Emerg Med. 2009;36:176-180.

6. Lee MJ, Park YG, Kim SJ, Lee JJ, Bang OY, Kim JS. Characteristics of stroke mechanisms in patients with medullary infarction. Eur J Neurol. 2012;19:1433-1439.

7. Edlow BL, Hurwitz S, Edlow JA. Diagnosis of DWI-negative acute ischemic stroke: a meta-analysis. Neurology. 2017;89:256-262.

8. Kattah JC, Talkad AV, Wang DZ, Hsieh YH, Newman-Toker DE. HINTS to diagnose stroke in the acute vestibular syndrome: three-step bedside oculomotor examination more sensitive than early MRI diffusion-weighted imaging. Stroke. 2009;40:3504-3510.

9. Seo MJ, Roh SY, Kyun YS, Yu HJ, Cho YK. Diffusion weighted imaging findings in the acute lateral medullary infarction. J Clin Neurol. 2006;2:107-112. 